\title{
PNEUMOCYSTIS JIROVECII INFECTION OF THE EXTERNAL AUDITORY CANAL
}

\author{
Catherine Mary Samuel, $M B C h B$ \\ Division of Medical Microbiology, University of Cape Town \\ Sairita Maistry, BSc, BSc (Hons), MB ChB, Dip Forensics (SA) \\ Division of Forensic Pathology, University of Cape Town \\ Estie Meyer, $M B$ ChB, FCORL (SA) \\ ENT Department, University of Cape Town \\ Andrew Whitelaw, MB BCh, MSc, FCPath (Micro) (SA) \\ Division of Medical Microbiology and National Health Laboratory Service, University of Cape Town
}

Pneumocystis jirovecii is well known to cause interstitial plasma cell pneumonia in immunocompromised patients. It has been implicated as a rare cause of infections in other anatomical sites. ${ }^{1}$ We report a rare case of P. jirovecii infection of the external auditory canal. This was the first manifestation of a previously unknown HIV infection.

\section{CASE REPORT}

A 27-year-old man was referred to the ENT clinic with a mass in the right external ear canal. He had no past medical history of note. Presenting symptoms included bloody otorrhoea, otalgia and decreased hearing in the right ear for 2 months. On examination the presence of a large polyp was confirmed. The tympanic membrane could not be visualised owing to the size of the polyp. The left ear appeared normal. The biopsy showed inflamed, partly necrotic granulation tissue, reported as a pyogenic granuloma. A trial of steroid-antibiotic ointment was commenced.

The patient's symptoms persisted, and he was booked for an examination under anaesthesia. Clinically he was pyrexial, with worsening otitis externa and a recurring polypoid mass formation with large, pale granulations. Biopsy resulted in profuse bleeding. The differential diagnosis was tuberculosis or glomus tympanicum. Laboratory investigations revealed an erythrocyte sedimentation rate of $60 \mathrm{~mm} / \mathrm{h}$ but a normal peripheral white cell count. He had no clinical evidence of pulmonary disease and a chest radiograph appeared normal. There was no other apparent source of infection. Histological examination revealed an acute inflammatory response with no granulomas, and a Ziehl-Neelsen stain was negative. An HIV test showed the patient to be HIV infected. Anti-tuberculosis (TB) medication was commenced empirically and a repeat biopsy was taken. The patient's pyrexia resolved and he was subsequently discharged.
The patient was lost to follow-up and presented again 3 months later. A necrotic polyp occluding the external ear canal was still evident. Biopsy taken at the previous presentation suggested a possible cholesteatoma. All specimens sent for TB culture were negative. The CD4 count from the previous admission was 24 cells/ $\mu \mathrm{I}$. An urgent temporal bone computed tomography (CT) scan revealed opacifications in the middle ear and mastoid cavity with the ossicles and scutum still intact, making the diagnosis of cholesteatoma less likely. The most recent biopsy showed granulation tissue, suppurative inflammation, focal giant cells, fibrin and copious amounts of foamy exudates. Special stains were performed to exclude other pathologies. Ziehl-Neelsen and Brown and Brenn stains were again non-contributory, but a Grocott-Gomori methenamine-silver stain (Fig. 1) showed the presence of Pneumocystis cysts confirming a diagnosis of otic pneumocystosis. The patient was commenced on high doses of oral trimethoprim-sulfamethoxazole and referred to the HIV clinic for initiation of highly active antiretroviral therapy. During a telephonic conversation 2 months later he reported symptomatic improvement. The otorrhoea and pain had subsided but he still had hearing loss.

\section{DISCUSSION}

Pneumocystis jirovecii infection is an important opportunistic infection. The organism was discovered in 1909 by Chagas, but the cystic forms were mistakenly considered to be part of the trypanosome life cycle. It was named P. carinii (Greek: pneumon 'lung', kystis 


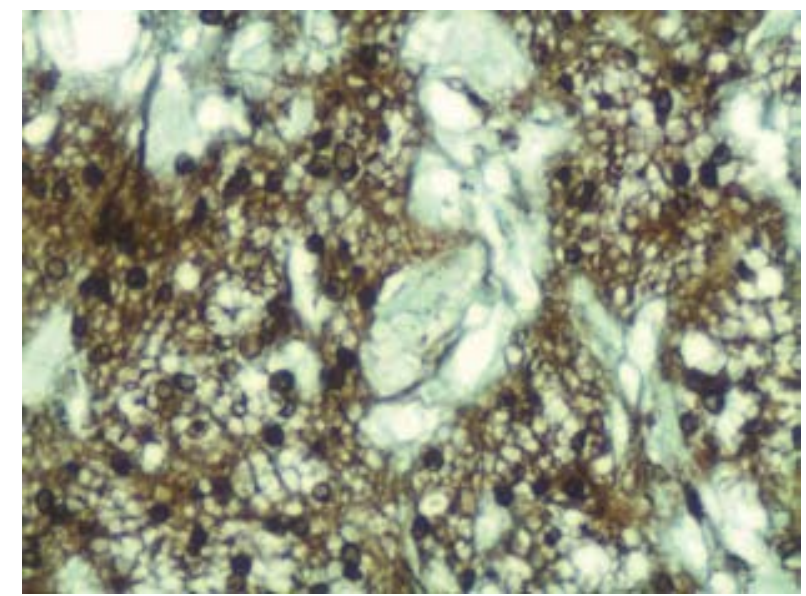

Fig. 1. Grocott-Gomori methenamine-silver stain showing Pneumocystis cysts of variable shape and size (40 $\times$ magnification).

'cyst'). ${ }^{2}$ Until 1988 the organism was considered to be a protozoan. The controversy regarding its taxonomy and classification continued for decades and recently, based on ribosomal RNA homologous to that found in fungi, ${ }^{2}$ it was re-classified as a fungus. Advances in molecular technology led to Pneumocystis organisms infecting humans being re-named $P$. jirovecii in 1999. ${ }^{3}$

P. jirovecii infection is an important opportunistic infection, and the incidence of pneumocystis pneumonia is on the increase in sub-Saharan Africa. Extrapulmonary pneumocystosis is, however, a rare complication and represents less than $1 \%$ of all cases of infection. ${ }^{4}$ One or more tissue or organ sites may be involved, with the most common being lymph nodes, bone marrow, spleen and liver. Otic pneumocystosis is exceedingly rare. It has only been described in the past 2 decades, and up to 2008 only 14 cases had been reported in the English literature. $^{3}$

It is postulated that under favourable conditions extrapulmonary dissemination can occur via the lymphatic and haematogenous routes. ${ }^{4}$ One theory is that these individuals may have more virulent strains. Observations in rat models suggest that heavy organism load and destruction of lung tissue may contribute to extrapulmonary dissemination. ${ }^{5}$ Conversely, $P$. jirovecii infection may be a systemic disease that most commonly affects the lungs. Extrapulmonary infection becomes more evident when prophylaxis is focused on the respiratory tract, and this may explain the correlation with the use of aerosolised prophylactic pentamidine. ${ }^{1,4}$

Although the mechanism of primary otic pneumocystosis is as yet unclear, it has been suggested that it may follow airborne invasion by trophozoites of the external auditory canal ${ }^{6}$ and that the middle ear may become involved after spread from nasopharyngeal carriage via the eustachian tube.

Individuals with otic pneumocystosis may have nonspecific symptoms of otorrhoea, otalgia, hearing loss, vertigo and tinnitus. Clinical findings include a mass in the external auditory canal and occasional otitis media. Destruction of the ossicles and sclerosis of the mastoid air cells, extensive bony erosion, and extension into the middle cranial fossa are rare complications documented in the literature. ${ }^{3}$

Diagnosis can be confirmed by biopsy, which is essential to rule out malignancy or other infective pathogens. ${ }^{6}$ Organisms can be demonstrated on microscopic examination of tissue sections and cytological preparations using silver, fluorescent or immunoperoxidase staining methods. The standard method using a Grocott-Gomori methenamine-silver stain is easy and reliable. Cystic forms are round to oval, 4 - $7 \mu \mathrm{m}$ in dimension, and appear as collapsed crescent- or helmet-shaped forms. The granulomatous reaction is composed of trophozoites and cysts, which appear as basophilic dots on HEtE. There is a variable inflammatory infiltrate.

As for pulmonary pneumocystis, medical therapy consists of oral or intravenous trimethoprim/sulfamethoxazole. Other regimens described include dapsone/trimethoprim, clindamycin/primaquine, atovaquone and pentamidine isethionate. ${ }^{1,3}$ Although there is usually resolution of symptoms after medical therapy, surgical excision of lesions in the external auditory canal may be clinically indicated.

Disseminated pneumocystosis, like Pneumocystis pneumonia, has high mortality rates. The prognosis may be better for those with single extrapulmonary site involvement and no concomitant lung infection. ${ }^{4}$ It is important for health care professionals to be aware of this infection as the HIV pandemic continues and results in common infections manifesting in extraordinary ways and in unusual sites.

\section{REFERENCES}

1. Ng VL, Yajko DM, Hadley WK. Extrapulmonary pneumocystosis. Clin Microbiol Rev 1997; 10(3): 401-418.

2. Dail DH, Tomashefski JF, Cagle PT, et al. Dail and Hammar's Pulmonary Pathology. 3rd ed. New York: Springer, 2008: 487-508.

3. Mahlakwane MS, Ramdial PK, Sing Y, et al. Otic pneumocystosis in acquired immune deficiency syndrome. Am J Surg Pathol 2008; 32(7): 1038-1043.

4. Raviglione MC. Extrapulmonary pneumocystosis: The first 50 cases. Rev Infect Dis 1990; 12(6): 1127-1138.

5. Chary-Reddy S, Graves DC. Identification of extrapulmonary Pneumocystis carini in immunocompromised rats by PCR. J Clin Microbiol 1996; 34: 1660-1665.

6. Breda SD, Hammerschlag PE, Gigliotti F, et al. Pneumocystis carinii in the temporal bone as the primary manifestation of the acquired immunodefiency syndrome. Ann Otol Rhinol Laryngol 1988; 97: 427-431. 